\title{
The Effect of the Relative Motion of Atoms on the Frequency of the Emitted Light and the Reinterpretation of the Ives-Stilwell Experiment
}

\author{
C.I. Christov
}

Received: 19 February 2009 / Accepted: 18 January 2010 / Published online: 29 January 2010

(C) The Author(s) 2010. This article is published with open access at Springerlink.com

\begin{abstract}
We examine the process of the emission of light from an atom that is in a relative translational motion with respect to the medium at rest in which the electromagnetic excitations propagate. The effect of Lorentz contraction of the of electron orbits on the emitted frequency is incorporated in the Rydberg formula, as well as the emitter's Doppler effect is acknowledged. The result is that the frequency of the emitted light is modified by a factor that is identical with what is called the 'relativistic Doppler effect'. The new emission formula is applied for reinterpretation of the Ives-Stilwell experiment and shown that within the second order of approximation with respect to the speeds of the atom and the 'absolute speed' (Earth's speed relative to the medium), the absolute motion does not affect the interference. The expression for the modification of the frequency involves both a first and a second-order term with respect to the speed of the atoms in the cathode tube. The latter turns out to be quantitatively the same as if the time would have changed its rate in the frame moving with the atoms. Thus, a new interpretation of the results of this famous experiment is provided without stipulating time dilation.
\end{abstract}

Keywords Preferred frame · Ives-Stilwell experiment - Emission of light · Earth's speed $\cdot$ Local standard of rest · Metacontinuum

\section{Introduction}

Following the discoveries of Young, Huygens, and Fresnel, the wave aspect of light was firmly established; however, the question then arose as of what was the medium that was 'waving'. Cauchy (see [1]) proposed that the luminiferous medium be considered as an elastic continuum. He introduced in the process the concept of stress

C.I. Christov ( $\varangle)$

Department of Mathematics, University of Louisiana at Lafayette, Lafayette, LA 70504, USA

e-mail: christov@louisiana.edu 
tensor, which is crucial to the modern mechanics of continua. In Cauchy's time, it was difficult to imagine particles 'plowing' through an elastic solid. For this reason, Kelvin and Stokes (and to a certain extent Maxwell) argued that the medium must be a thin fluid-like substance, which was called the 'ether'.

The continuum concept emerged with even greater urgency after Maxwell formulated the equations of electrodynamics [2], and Hertz [3] later provided the experimental verification of the existence of electromagnetic waves of a specific frequency range. The notion of a field epitomizes, essentially, the same meaning as continuum, and presumes a set of spatially distributed interacting infinitesimal points.

Concerning the detection of the absolute medium, a great deal of hope was attached to the experiment outlined by Maxwell, in which a light beam is split and sent on two different paths with consecutive interference of the two beams. Michelson [4] and then Michelson and Morley [5] achieved a virtuoso implementation of Maxwell's proposal using an experimental scheme based on the Michelson interferometer. This is now known as the celebrated Michelson and Morley Experiment (MME). However, the hopes to measure Earth's speed with respect to the absolute continuum evanesced when MME turned a nil result. Subsequently, the MME was thoroughly evaluated and it was re-established by other investigators, and a discussion then ensued of how to interpret the nil result. It was eventually agreed to be considered as a 'proof' that there exists no absolute medium where light propagates. This conclusion is hailed as one of the most important conceptual achievements of modern physics, because it led to a precipitous paradigm shift: the mechanistism of the nineteen century was replaced by a more abstract world view.

The problem with concluding that the luminiferous medium does not exist is that such a conclusion is overreaching, and as such it presents a logical fallacy. Actually, the only rigorous conclusion which can be drawn from the nil result of MME is that the absolute continuum cannot be detected by this particular experiment. This less overreaching conclusion, which satisfies also Occam's razor, is embodied in the proposition of Fitzgerald (see, [6, p. 749]) and Lorentz [7], who explained the nil result by the possibility of length contraction in the direction of motion. Indeed, in a Universe where lengths shorten in the direction of motion, a MME type of experiment is, in principle, not capable of detecting the absolute continuum. It should be noted that MME is based on what can be called 'phase-shift' interferometry (see the overview in [8]), in which the effect sought is exactly cancelled by the supposed contraction of lengths. Clearly, accepting the contraction assumption instantly rendered the dismissal of the absolute medium superfluous. Thus, "the baby was thrown out with the bath water", but the logical inconsistency did not seem to attract much scrutiny. The likely reason for this was that the scientists of the time believed that there were other reasons to stick with the idea (iconoclastic for its time) that there was no material medium in which light propagates.

It should be stressed here that the Lorentz-Fitzgerald contraction has been splendidly confirmed in all major experimental tests, and can be considered now as one of the most important discoveries in physics. It is equally valid with or without an absolute continuum. (Actually, both Fitzgerald and Lorentz believed that the contraction in the direction of motion was the reaction to the presence of an ether.) The logical inconsistencies arose when researchers tried to read too much in the fact that lengths 
contract in the direction of motion, attributing the contraction to some abstract, hitherto unaccounted for properties of space and time, which cannot be explained by continuum mechanics.

A little earlier, before the contraction hypothesis was proposed, Voigt, Larmor, and Lorentz [7, 9, 10] introduced what is nowadays known as the Lorentz transformation (LT), in order to make the wave equation invariant in a moving frame. In LT, time is demoted from its absolute status and is obligated to change in such a manner that leaves the linear wave equation invariant in the moving frame. One of the predictions from the LT was that lengths must contract in the direction of the motion. The latter was considered as an important success of LT. The impact of LT on physical thought prompted Poincare to propose the "Relativity Principle" (RP). One of the formulations of RP claims that the non-accelerating motion of a frame cannot be detected. Thus, LT was the main inspiration of what is now called the 'Special Relativity Theory' (see the original work [11] and the review [12]).

Another important discovery of modern physics took place three decades ago when the anisotropy of the cosmic microwave background radiation (CMBR) was discovered. This finding clearly defined the speed of Earth as being of order of several hundred kilometers per second (see [13, 14]). Currently, there seems to be a consensus that not all inertial frames are created equal, and that one should consider, at least locally, a preferred frame. A good overview of the situation and the challenges that the accepted point of view is still facing can be found in [15]. It is clear that regardless of the terminology ('preferred frame' [15], 'physical vacuum' [16], 'Local Standard of Rest' (LSR) [14]), there is a field (in the sense of a continuous structure) and that our speed relative to it is well defined. The presence of an anisotropy in the CMBR flies directly in the face of the postulate that the speed of an inertial frame, which is uniformly translating, cannot be measured from within the frame.

In the Nineteenth century, it was believed that the preferred frame is connected with a substance pervading the empty geometrical space. Since this substance was not found to obstruct the motions of material bodies, it was assumed to be a thin, 'ethereal', substance. A radically different approach has been proposed by the present author, in which space itself is considered as a mechanical continuum. It is clear that any kind of forces acting between different points of a material space are the result of the internal stresses of the mechanical continuum. The mechanical continuum is distinguished from the empty vessel called 'geometrical space' by the internal stresses acting between the points, as well as by the time derivatives, which are no longer the partial time derivative, but frame-indifferent derivatives, which are generalizations of Euler's convective derivative. In [17, 18], it was shown that for the Maxwell displacement current, the proper frame-indifferent time derivative is the Oldroyd [19] upper-convected derivative. What is more, the new formulation of the electrodynamics presented in $[17,18,20]$ was shown to be Galilean invariant. Actually, the frame-indifference is the proper generalization of Galileo's principle (formulated for a single material point/particle) to a system of interacting material points (i.e., a continuum). As a result, the Lorentz invariance was replaced by what is called 'material invariance' (frame indifference), which is true for any accelerating and/or deforming frame. This prompted the author to introduce the 'Material Invariance Principle' (MIP) in lieu of RP in [17]. The detailed derivations concerned with the notion of 
frame indifference for the material space can be found in a recent paper [20], in which it was also shown that the new formulation incorporates the Lorentz-force and the Biot-Savart law in the governing equations of the model. This means that MIP has two main advantages over RP: (i) it is valid in any accelerating and/or deforming frame; (ii) it unifies all the known laws of electromagnetism in a single theory, which generalizes that of Maxwell [17, 18, 20].

The important difference of this new model of mechanical space (referred to as metacontinuum [21]), as compared to the old aether theories, is that the particles and charges are now considered as phase patterns, i.e., solitons, that propagate over the metacontinuum, rather than plowing through it. This alleviates the problem connected with the existence of an 'aether wind': a phase pattern does not disturb the integrity of the underlying substrate over which it propagates. In $[18,20]$, the charges were interpreted as the torsional localized waves of the metacontinuum, which necessarily undergo Lorentz contraction when propagating (a well known fact from the theory of solitons). Thus, one more experimentally established fact is explained by the MIP in a more consistent fashion than RP, without invoking an additional hypothesis, such that the Universe is Lorentz invariant. In MIP, the constancy of speed of light, the material invariance, and the Lorentz contraction are intrinsic properties of the model. The model of mechanical space turned out to be able to also explain the master equation of wave mechanics (i.e., Schrödinger's equation see [21, 22]), if the metacontinuum is considered as a thin 3D layer suspended in the four dimensional geometric space. The equation for the flexural deformations of this shell-like construct is nothing else but the Schrödinger equation, if written for the real part of the wave function. In addition, the concept of particles being solitons (or what is called quasi-particles) predicted an attractive force proportional to the inverse square of the distance between two solitons (quasi-particles) [22]. All this, clearly, lends additional support to the idea of the mechanical space.

The above outlined logical fallacy in the interpretation of MME does not necessarily invalidate the assumptions that led to relativity, since one of the explanations of the nil effect is indeed the possibility that a material substrate, of the known types of continuous media, does not actually exist. Yet, it is scientifically not satisfactory to adopt such a far-reaching conclusion without exhausting all the possibilities to measure the absolute speed. ${ }^{1}$ This logical inconsistency calls for the reexamination of other experiments believed to confirm that an absolute continuum cannot exist. In [23, 24] this author has proposed a new approach to the interferometry measurement based on beat frequency, rather than on phase, as in MME experiment. Unfortunately, such kind of beat-frequency measurement has yet to be implemented. Consequently, it is very important to re-examine the available experimental results, which were believed to have provided the proof for RP.

A most important experimentum crucis is the seminal work of Ives and Silwell $[25,26]$ (ISE), which is commonly accepted to have proved the reality of time dilation. The above analysis of the logical and mathematical tenability of MIP or RP necessitates a closer look at the way we are trying to detect the absolute motion (the motion relative to the absolute continuum). How the emission of light is influenced

\footnotetext{
${ }^{1}$ The 'absolute' speed is the speed of a frame relative to the resting absolute medium.
} 
by the translation of matter relative to the supposed absolute continuum in which light propagates, has never been examined with the necessary precision and scrutiny. Here we propose a new point of view on the subject, which indicates that the Ives-Stilwell experiment (ISE) is another one that can be well explained without recourse to the Lorentz transformation and time dilation.

\section{Emission of Light by Moving Atoms}

A photon is emitted/absorbed when an electron jumps from one orbit to another. If the atom is at rest with respect to the absolute continuum, then for the particular jump, the frequency is defined by the distance between the orbits. If so, the frequency of an emitted photon is governed by the Bohr-Rydberg law

$$
\omega_{\mathrm{rest}}=c R\left(\frac{1}{n_{f}^{2}}-\frac{1}{n_{i}^{2}}\right)=\alpha \frac{c}{r}\left(\frac{1}{n_{f}^{2}}-\frac{1}{n_{i}^{2}}\right)=\alpha c\left(\frac{1}{r_{f}}-\frac{1}{r_{i}}\right)=\alpha c \frac{r_{i}-r_{f}}{r_{f} r_{i}},
$$

where $n_{f}, n_{i}\left(n_{i}>n_{f}\right)$ are the numbers of the respective orbits between which the electron jumps, and $\alpha=e^{2} /(\hbar c) \approx 1 / 137$ is the fine structure constant. Respectively,

$$
R=\frac{2 \pi^{2} e^{4} m}{c h^{3}} \mathrm{~cm}^{-1} \approx 109737 \mathrm{~cm}^{-1}, \quad r=\frac{h^{2}}{4 \pi^{2} m e^{2}} \approx 0.528 \cdot 10^{-8} \mathrm{~cm},
$$

are the Rydberg number and the atomic radius (see e.g. [27, Chap. 39]). Then $r_{f}=$ $r n_{f}^{2}$ and $r_{i}=r n_{i}^{2}$.

The question now is how the emitted frequency is influenced by the fact that the atom is moving with respect to the supposed absolute medium. The problem was first addressed by Larmor $[10,28]$ who argued that the motion of the atom can lead to the increase of the period of the electron orbiting the proton, and hence can lead to apparent 'time dilation' (see also the illuminating discussion in [29, Chap. 9]). Here we focus on the effect of the shortened dimensions of the moving atom. As mentioned in the introduction, our approach is based on two principles:

1. Space is a mechanical continuum whose points are connected to each other because of the continuity and are interacting via the internal stresses.

2. What is perceived as particles and charges are, actually, nonlinear localized waves (solitons or 'quasi-particles') of deformation.

As shown in $[18,20]$, any phase pattern in the metacontinuum is shortened in the direction of propagation (motion). Thus, the Lorentz contraction is a corollary here, not an independent assumption. Also, the speed of light is absolute, because it is the characteristic speed of linear shear waves in a continuum, and has nothing to do with the translational speed of the rectilinear propagation of different phase patterns.

We can consider the atom as being a translating phase pattern (quasi-particle), whose dimensions are shortened in the direction of motion. This means that the orbits of electrons become curves on ellipsoids the length of whose shortest axis is proportional to the inverse of the Lorentz factor. That is, the shortest axis of the ellipsoid that includes a particular axis is $r=r_{\text {rest }} \sqrt{1-w^{2} / c^{2}}$, where $w$ is the speed 
of the relative motion of the phase pattern (identified as the atom) with respect to the absolute continuum (the 'absolute speed'). Thus, $r$ is the length scale that defines the largest energy of the electron while orbiting the proton. Since the emitted frequency is proportional to the inverse of the atomic radius $r$, the frequency emitted by an atom whose characteristic length is shortened by the Lorentz contraction is given by

$$
\omega_{\text {contracted }}=\frac{\omega_{\text {rest }}}{\sqrt{1-w^{2} / c^{2}}} .
$$

A similar expression to (3) for the frequency can be obtained, from the Larmor formula for shortening the orbital time of the electron (see the enlightening exposition in [30] and the original work [10, p. 229]). While qualitative the same, the Larmor's derivation does not answer the question what is the relevance of the orbital time to the actual frequency of the emitted photon.

At this junction, it is to be pointed out that the absolute speed has not been measured so far. The important thing to understand here is that the photon is emitted by the atom, but detaches from it and lives on its own as a shear wave in the absolute continuum. The photon is not concerned with whatever frame the observer may chose to associate with the moving atom. Then the emitter's Doppler effect applies to the emitted wave making the frequency dependent on the direction of propagation of the wave front (the direction of the ray), namely

$$
\omega_{\mathrm{mov}}=\frac{\omega_{\mathrm{rest}}}{\sqrt{1-w^{2} / c^{2}}}\left(1+\frac{w}{c} \cos \theta\right),
$$

where $\theta$ is the angle between by the ray and the direction of translation of the atom. For the frequency of the waves propagating in the positive and negative directions of the translation of the atom $(\theta=0, \pi)$ we get

$$
\omega_{\mathrm{mov}}^{ \pm}=\omega_{\mathrm{rest}} \frac{\sqrt{1 \pm w / c}}{\sqrt{1 \mp w / c}} .
$$

Equation (4a) is exactly the one derived by Einstein [11, §7] based on the LT. Note that he speaks of the observer's effect, while here we have the emitter's effect. The main difference, however, is in the interpretation. In the argumentation of the relativistic Doppler effect, the relative speed of the two frames is assumed to cause time dilation of the observer [31, p. 6, 32, Chap. 5]. In our interpretation, we obtained the same formula, assuming that the time is absolute, and just accounting for the effect of Lorentz contraction on the Bohr-Rydberg formula for the emitted frequency. This lends some new support to the Lorentz point of view (spatial contraction, but no time dilation), which was dismissed many years ago without actually examining the full effect of the contraction of moving atoms, the way it is done in the present paper.

A sketch of the contraction of the atom is presented in Fig. 1. The Doppler effect is illustrated for the case when a photon jumps against the motion $(\theta=\pi$ in (4a)).

It is important to note here that in (4) enters the absolute speed. If both the emitter and the observer are moving with respect to the absolute medium with relative speed to each other considerably lesser than their absolute speeds, then the contraction is 
Fig. 1 Contraction of the emitting atom in the direction of translation (solid lines). Dashed lines represent the atom in a moment of time needed for the photon to jump from one orbit to the other

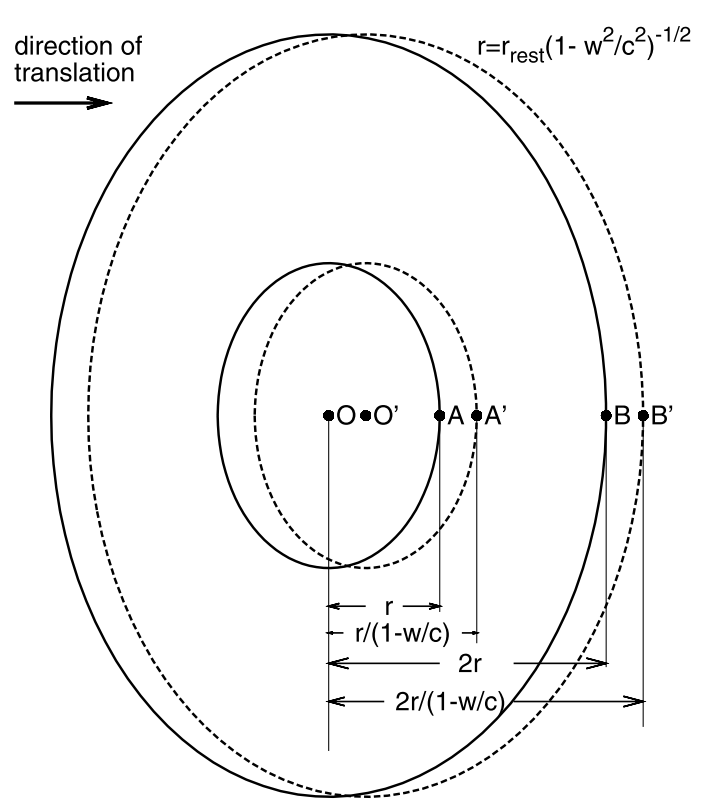

mainly defined by the absolute speed, and the emitter's and the receiver's Doppler effects will be superimposed (as in the relativity theory). In any case (even for rather slow absolute speed of one of the frames with respect to the relative speed), there will be a difference, albeit small, in the frequencies as predicted by the present work or by the use of the relativistic counterpart of (4). Yet, it will be still there, and it should be possible to find the vestiges of the absolute speed in experiments involving interference. The only issue is what is the order of the effect connected with the absolute speed. A situation in which it may be possible to contra-distinct between the relativistic and absolutistic versions of (4) is at hand in the seminal Ives-Stilwell experiment.

\section{Ives-Stilwell Experiment}

In the ISE, atoms are emitted in a cathode tube and the light from the moving atoms interferes with the light from the atoms that are at rest with respect to the experimental frame (cathode tube). Clearly, the photons that interfere in ISE can be emitted both in the direction of the absolute motion or against it. According to the above derivations, the frequencies of the waves emitted by the speeding atoms and of the atoms at rest are respectively

$$
\omega_{e}=\omega \frac{\sqrt{(1+u / v)}}{\sqrt{(1-u / c)}}, \quad \omega_{r}=\omega \frac{\sqrt{(1+v / c)}}{\sqrt{(1-v / c)}},
$$

where we have used (4b) with $w=u$, and $w=v$, respectively. Here $v$ is the unknown component of Earth's absolute speed that is parallel to the axis of the cathode tube, 
and $u=v+q$ is the unknown component of the velocity of the moving atoms with respect to the absolute medium. The quantity $q$ is the known speed of the atoms relative to the cathode tube. Note that the signs of $u, v$ are a matter of convention and we assume the positive sign. Now, the sign of $q$ is not trivial: it is positive for the photons emitted from the atoms moving in one direction (say the direction parallel to the component of the absolute speed along the axis of the cathode tube), and negative for the photons emitted by the atoms moving in the opposite direction (antiparallel to the axis of the cathode tube). Let us introduce a small parameter $\varepsilon=\max (|u / c|,|v / c|,|q / c|)$. Using the software package Mathematica (version 6.0), one can expand the ratio of the two frequencies from (5), thus yielding

$$
\frac{\omega_{e}}{\omega_{r}}=\frac{\sqrt{1-v / c} \sqrt{1+q / c+v / c}}{\sqrt{1+v / c} \sqrt{1-q / c-v / c}}=1+\frac{q}{c}+\frac{q^{2}}{2 c^{2}}+\frac{q^{3}}{2 c^{3}}+\frac{q^{2} v}{c^{3}}+\frac{q v^{2}}{c^{3}}+O\left(\varepsilon^{4}\right) .
$$

Within the order of approximation $o\left(\varepsilon^{2}\right)$, one gets for the observed frequency shift (the beat) of the moving atoms, the following:

$$
\frac{\Delta \omega}{\omega_{r}} \equiv \frac{\omega_{e}-\omega_{r}}{\omega_{r}}=\frac{q}{c}+\frac{1}{2} \frac{q^{2}}{c^{2}} .
$$

In terms of the wavelength, within the same order of approximation, one has

$$
\frac{\Delta \lambda}{\lambda}=-\frac{q}{c}+\frac{1}{2} \frac{q^{2}}{c^{2}} .
$$

The last formula shows that the absolute speed of Earth, as parameterized by $v$, does not affect the final result within the second order. There is a first order Doppler effect connected with the relative speed $q$ of the atoms in the cathode tube, and it is of different sign for the forward and backward emitted waves (positive or negative $q$ ). (Such 'splitting' of the spectral lines was observed in [25].) After the subtraction of the first order for the backward and forward waves, the net outcome of ISE within the order $O\left(\varepsilon^{2}\right)$ is the second order term $\frac{1}{2} q^{2}$, which is quantitatively the same as the one predicted by the hypothesis of time dilation. The experimental data was compared to the prediction from the hypothesis of time dilation and shown in [26] to be in a very good quantitative agreement.

Now, the standard treatment based on the special theory of relativity would have in the third order only the term $\frac{1}{2} q^{3} / c^{3}$ in (6). The hypothesis of the absolute continuum shows non-trivial third-order terms containing $v$ in (6), and the latter can give the opportunity to distinguish one theory from the other. The problem is that the cubic terms are very small, and what aggravates the situation is that the terms containing the absolute speed, $v$, are smaller than the term containing the speed $q$ of the atoms in the cathode tube. Indeed, from the LSR measurements we can estimate $v / c \approx 10^{-3}$, while $q / c$ can be as high as $4 \cdot 10^{-3}$. This means that the dominant third order term in (6) will be $\frac{1}{2} q^{3} / c^{3}$ for the speeds of the atoms involved in $[25,26]$. One way to increase the relative importance of $v$ is to repeat the ISE with smaller speeds (i.e., smaller voltages), but then the sensitivity may not be enough. The other approach is to go for even higher $q$ 's, so the overall magnitude of the third-order effect is 
increased. Simultaneously, the sensitivity must be increased, so that the relatively smaller contribution from $v q^{2}$ can be reliably extracted from the overall third-order effect. This makes carrying out an IS type of experiment quite a challenge when the absolute speed is targeted. Clearly, the search for first-order experiments, such as the one described in [24], should continue.

It is important to understand that the first-order term detected in the ISE already supports the notion of existence of a material substrate. However, the absence of dependence on the Earth's speed in the ISE was considered yet as another proof that the absolute medium does not exist, and that the only explanation of the term $\frac{1}{2} q^{2}$ is the time dilation. In the present paper, we have shown that an alternative explanation of the ISE is possible, hence the jury is still out on time dilation as a property of our particular Universe.

\section{Time Dilation or Frequency Change?}

Absolute motion is very elusive, indeed. It cannot be detected, in principle, by an experiment of the above type (ISE), nor by MME if merely the second-order effect is measured. Thus, it comes as no surprise that it hasn't been detected in a laboratory setting. The result presented here allows one to claim that the hypothesis of existence of an absolute luminiferous medium is compatible with the results of IvesStilwell experiment, and it should be given the proper consideration alongside with the hypothesis of time dilation. This conclusion is in agreement with the fact that electrodynamics can be regarded as the manifestation of the internal stresses in an absolute continuum (called metacontinuum in [17, 18, 20, 21]).

In this work, we have examined the emission of light from an atom that is in a rectilinear translatory motion relative to the medium where the electromagnetic waves propagate. A modification of the Bohr-Rydberg formula is proposed for the frequency of the emitted photon, when the translatory motion of the atom is accounted for. The new formula has the same analytical expression for the frequency within the second order of the relative speed of the atoms as the formula for the relativistic Doppler effect. It can be called the 'intrinsic Doppler effect' (IDE) for the emission of light by moving atoms.

Furthermore, the IDE derived in the present work has been used to reexamine one of the experimenta crucis: the ISE. We have shown that IDE explains the observed changes of frequency in cathode tubes. Since in the present context, there is no way to determine reality of the time dilation, apart from showing changes in frequency, the result of the ISE related to the frequency cannot be considered as an unequivocal proof of the non-existence of the absolute continuum, and the inevitability of time dilation. The latter can only be considered as one of the possible explanations of the frequency change along with the formulation provided in the present paper.

\section{Conclusions}

The existence of an 'Intrinsic Doppler effect' (IDE) is shown in the present paper based on the assumption that there exist an absolute medium (field) whose properties 
are not influenced by the moving matter. The re-interpretation of ISE presented here can be considered as one of the possible verifications of such a hypothesis. We have shown that the relative translation of a frame cannot be detected within the frame up to terms of second order, but not because some principle forbids it (called Relativity Principle), but because of the nature of the emission of light from moving atoms. Only when the third-order effects are examined, and if they do not comply with the result of the present work, one can say that another hint that no absolute continuum exists has been found.

Acknowledgements This research has been supported in part by a grant from Louisiana Board of Regents through contract NSF(2009)-PFUND-156. The author is indebted to the anonymous referees for the very helpful and crucial suggestions.

Open Access This article is distributed under the terms of the Creative Commons Attribution Noncommercial License which permits any noncommercial use, distribution, and reproduction in any medium, provided the original author(s) and source are credited.

\section{References}

1. Whittaker, E.: A History of the Theories of Aether \& Electricity, vols. 1 and 2. Dover, New York (1989)

2. Maxwell, J.C.: Philos. Trans. R. Soc. Lond. 155, 469 (1865)

3. Hertz, H.: Electric Waves. MacMillan, London (1900)

4. Michelson, A.A.: Am. J. Sci. 22, 120 (1881)

5. Michelson, A.A., Morley, E.W.: Am. J. Sci. 34, 333 (1887)

6. Lodge, O.: Philos. Trans. R. Soc. Lond. A 184, 727 (1893)

7. Lorentz, H.A.: In: The Principle of Relativity, pp. 3-7. New York (1952)

8. Tolansky, S.: An Introduction to Interferometry. Wiley, New York (1955)

9. Ernst, A., Hsu, J.P.: Chin. J. Phys. 39(3), 211 (2001)

10. Larmor, J.: Philos. Trans. R. Soc. 190, 205 (1987)

11. Einstein, A.: Ann. Phys. 891-921 (1905)

12. Einstein, A.: Relativity. The Special and the General Theory. Three Rivers Press, New York (1961)

13. Corey, B.E., Wilkinson, D.T.: Bull. Astron. Astrophys. Soc. 8, 351 (1976)

14. Smoot, G.F., Gorenstein, M.V., Miller, R.A.: Phys. Rev. Lett. 39, 898 (1997)

15. Consoli, M., Costanzo, E.: Phys. Lett. A 333, 355 (2004)

16. Lee, T.D.: Nucl. Phys. A 553, 3 (1993)

17. Christov, C.I.: Found. Phys. 36, 1701 (2006)

18. Christov, C.I.: Math. Comput. Simul. 74, 93 (2007)

19. Oldroyd, J.G.: Proc. R. Soc. A 200, 523 (1949)

20. Christov, C.I.: Nonlinear Anal. 71, e2028 (2009)

21. Christov, C.I.: In: Markov, K. (ed.) Continuum Models and Discrete Systems-Proceedings of CMDS8, pp. 370-394. World Scientific, Singapore (1996)

22. Christov, C.I.: Math. Comput. Simul. 80, 91 (2009)

23. Christov, C.I.: Prog. Phys. 3, 55 (2006)

24. Christov, C.I.: J. Opt. Soc. Am. A 26, 2292 (2009)

25. Ives, H.E., Stilwell: J. Opt. Soc. Am. 28, 215 (1938)

26. Ives, H.E., Stilwell: J. Opt. Soc. Am. 31, 369 (1941)

27. Joos, G.: Theoretical Physics, 2nd edn. Dover, New York (1986)

28. Larmor, J.: Aether and Matter. C.J. Clay and Sons, Cambridge (1990)

29. Bell, J.S.: Speakable and Unspeakable in Quantum Mechanics. Cambridge University Press, Cambridge (2004)

30. Macrossan, M.N.: Brit. J. Philos. Sci. 37, 232 (1986)

31. Gill, T.P.: The Doppler Effect. Logos Press, London (1965)

32. French, A.P.: Special Relativity. Norton, New York (1968) 